\title{
Proximidade geográfica e interação universidade-empresa no Rio Grande do Sul
}

\author{
Achyles Barcelos da Costa ${ }^{1}$ \\ Janaina Ruffoni ${ }^{2}$ \\ Daniel Puffal ${ }^{3}$
}

\begin{abstract}
Resumo: O objetivo deste trabalho é avaliar o papel que a proximidade espacial desempenha nas atividades inovativas de firmas no âmbito da interação entre universidades e empresas localizadas no estado do Rio Grande do Sul (RS). Foi feita investigação com dados secundários - Censo de 2004 do Diretório dos Grupos de Pesquisa do Conselho Nacional de Desenvolvimento Científico e Tecnológico (CNPq) e com dados primários de uma pesquisa survey em 2009 com empresas que os grupos de pesquisa informaram estabelecer interação. Resultados da investigação indicam que a proximidade geográfica importa e que a maior concentração $(43,5 \%)$ das interações observadas ocorre em um espaço territorial relativamente pequeno (até $50 \mathrm{~km}$ de distância), e que $2 / 3$ do total de interações dá-se com instituições sediadas no RS. Contudo, constatou-se que 21 empresas se relacionaram com universidades/instituições de fora do Estado, o que denota a existência de outras proximidades, além da geográfica, que também são importantes para as suas atividades inovativas.
\end{abstract}

Palavras-chave: Interação universidade-empresa; Proximidade geográfica; Rio Grande do Sul/Brasil.

\footnotetext{
1 Economista. Professor da Universidade do Vale do Rio dos Sinos (Unisinos).

E-mail: achyles@portoweb.com.br

2 Doutora em Política Científica e Tecnológica pela Universidade Estadual de Campinas (Unicamp). Professora do Programa de Pós Graduação em Economia da Universidade do Vale dos Sinos (Unisinos). E-mail: jruffoni@unisinos.br

3 Doutor em Administração pela Universidade do Vale do Rio dos Sinos (Unisinos). Professor na Universidade Feevale e na Instituição Evangélica de Novo Hamburgo (IENH). E-mail: puffal@micropol.com
} 


\title{
Geografic proximity and university- industry interactions in Rio Grande do Sul
}

\begin{abstract}
The purpose of this paper is to evaluate the role that spatial proximity plays in innovative activities of firms in the context of interaction between universities and business enterprises located in the State of Rio Grande do Sul (RS). Research was done with secondary data - census 2004 from Directory of Research Groups of $\mathrm{CNPq}$ - and with primary data from a survey in 2009 with enterprises that research groups have reported establishing interaction. Research results indicate that geographical proximity matters and the largest concentration (43.5\%) of the interactions observed occurs in a relatively small geographical area (up to $50 \mathrm{~km}$ away), and that 2/3 of total interactions are with institutions based on $R S$. However, it was found that 21 companies interact with universities/ institutions outside the State, which denotes the existence of other proximity, in addition to geographical, that are also important to their innovative activities.
\end{abstract}

Keywords: University-industry linkages; Geographic proximity; Rio Grande do Sul/Brazil.

JEL: $O_{3}$.

Introdução

A compreensão dos elementos determinantes do progresso técnico de nações, setores produtivos e empresas tem sido objeto de discussão de estudiosos, principalmente dos denominados economistas evolucionistas e neo-schumpeterianos, e está longe de ser uma questão plenamente estabelecida. Entender o progresso técnico exige estudar o processo de geração e difusão de inovações. Uma forma de avançar nessa percepção é examinar como ocorre a interação entre diferentes agentes econômicos, sociais e institucionais. Especificamente a análise contempla as ligações estabelecidas entre universidades e institutos de pesquisa com empresas. Os elementos que influenciam e determinam as relações criadas entre essas instituições são diversos, bem como as características de tempo, envolvimento e resultados da interação. Uma importante questão que vem sendo discutida pela literatura é a influência que a proximidade geográfica exerce nesse processo. Assim, o objetivo deste trabalho é avaliar o papel que a proximidade espacial desempenha nas atividades inovativas de firmas no âmbito da interação entre universidades e empresas localizadas no estado do Rio Grande do Sul (RS). 
O estudo aqui apresentado faz parte de uma pesquisa mais ampla sobre interação universidade-empresa no Brasil que vem sendo realizada desde $2006^{4}$. O objetivo daquela pesquisa é o de identificar os padrões de interação existentes entre universidades e empresas no País.

A estrutura do trabalho é formada por esta Introdução e mais quatro seções. A segunda seção é dedicada a uma resenha teórica acerca da literatura sobre os determinantes da inovação em sua conexão com o conhecimento, o aprendizado interativo e o papel que a proximidade geográfica desempenha nesse processo. Ela serve como um organizador do conteúdo discutido nas seções seguintes. A terceira seção apresenta a metodologia empregada e as características gerais da amostra de empresas que constaram da pesquisa de campo. Na quarta seção é feito, então, um exame do material pesquisado sobre o papel que a proximidade geográfica tem no desempenho inovativo de empresas localizadas no Rio Grande do Sul. A quinta seção finaliza a análise com um apanhado geral da discussão e dos principais resultados alcançados.

\section{Conhecimento, aprendizado interativo, proximidade e inovação}

A geração e a difusão de conhecimentos e de inovações apresentam-se dinâmicas e com características complexas, envolvendo um conjunto diversificado de agentes, como as universidades e seus laboratórios, as empresas e suas estruturas formais e informais de pesquisa e desenvolvimento, bem como a ação de governos mediante regulação e com suas instituições públicas de fomento e de pesquisa ligadas a áreas específicas. Trata-se, enfim, da concepção, da transmissão e da absorção de diferentes tipos de conhecimentos que resultam da interação de organizações com culturas e objetivos distintos. Além disso, a interação ocorre tanto em um espaço geográfico próximo, localizado, quanto em âmbito mais distante, seja nacional ou internacional.

A justificativa de estudos na área de interação universidades/institutos de pesquisa e empresas (Interação U-E) é que esse mecanismo pode se constituir em importante canal de desenvolvimento tecnológico de empresas, regiões e países, proporcionando capacidade para o catching up tecnológico e o fortalecimento do desempenho econômico. Particularmente em países de menor desenvolvimento relativo, que se veem às voltas com escassez de

4 Pesquisa intitulada Interações de Universidades e Institutos de Pesquisa com Empresas no Brasil, coordenada pelo Prof. Dr. Wilson Suzigan [wsuzigan@pq.cnpq.br], do Departamento de Política Científica e Tecnológica (DPCT) da Universidade Estadual de Campinas (UNICAMP) e pelo Prof. Dr. Eduardo da Motta e Albuquerque, do Centro de Desenvolvimento e Planejamento Regional (CEDEPLAR) da Universidade Federal de Minas Gerais (UFMG). O projeto obteve recursos da FAPESP (Processo n ${ }^{\circ}$ 06/58878-8) e do CNPq (Auxílio Pesquisa ${ }^{0}$ 478994/2006-o). Atualmente acha-se aprovado o projeto "Padrões de Interação Universidade-Empresa no Brasil", submetido ao Edital MCT/CNPq 14/2009 - Universal - Faixa B. O presente trabalho insere-se também no Edital FAPERGS o06/2010. Programa Pesquisador Gaúcho. 
recursos, esse também é um mecanismo que permite às empresas viabilizar seu envolvimento em pesquisa e desenvolvimento (P\&D). No caso de universidades, essa prática possibilita que se mantenham em linha com as necessidades de formação de recursos humanos e de exploração de novas áreas do saber. No âmbito de políticas industrial e de inovação, o governo passa a contar com um leque ampliado de instrumentos para estimular o investimento e tornar as políticas públicas mais eficazes na geração de emprego e renda.

Para que as empresas inovem, contudo, é necessário ter acesso à informação e ao conhecimento. $\mathrm{O}$ conhecimento, de acordo com ampla literatura sobre o assunto, pode ser codificado ou tácito (Nelson e Winter, 1982; Gertler, 2003; entre outros). O primeiro é traduzido em manuais, instruções e códigos que são transmitidos através de símbolos e linguagem padrão. A sua aquisição pode, inclusive, ser feita de forma impessoal, através do mercado. Embora conhecimentos explícitos ou codificados possam ser transmitidos de forma rápida e a longas distâncias, nem sempre as empresas têm acesso a ele de forma facilitada. O mercado para esse tipo de transação pode apresentar imperfeições. A existência, por exemplo, de barreiras à entrada na forma de direitos de propriedade exclusivos (patentes), pode inibir o acesso de empresas a certos tipos de conhecimento e, assim, influenciar em sua capacidade inovativa.

O conhecimento tácito é aquele em que as informações a ele associadas não possuem um código ou uma linguagem comum em que possam ser transmitidas de forma facilmente inteligível, como ocorre com os sinais emitidos pelo sistema de preços. Nesses casos, como assinalado por Piore (2001), é necessária uma linguagem real que possa transmitir as informações requeridas necessitando, para tal, da presença física de seus agentes portadores (dado que o conhecimento tácito está neles incorporado) ou de intermediários sociais para que esse tipo de saber tenha sucesso na sua difusão.

São diversas as formas sob as quais as empresas podem ter acesso ao conhecimento. Um dos estudos pioneiros sobre o assunto, conforme Rosenberg ([1982] 2006) é o de Kenneth Arrow escrito em 1962, intitulado The Economic Implications of Learning by Doing, onde aquele autor ressalta que o aprendizado ocorre pela prática diária na manufatura de um produto: o chamado 'aprender fazendo' [learning-by-doing], como informa o título de seu artigo. Contudo, segundo ainda Rosenberg, existe outro tipo de aprendizado que decorre não da manufatura em si, mas da utilização do produto pelo usuário final [learning-by-using]. Isso se aplica àqueles casos em que a empresa adquire um novo bem de capital e cujo domínio exige tempo de uso. Entretanto, quando da fabricação desse bem, em um ambiente permeado pela divisão do trabalho, o produtor deveria conhecer as necessidades dos potenciais usuários, de modo que o projeto do produto atenda aos usos em que possa ser aplicado. A atomização de agentes e a decorrente impessoalidade de 
mercado não permitem conhecer esses requisitos, dado que os sinais emitidos nas trocas informam apenas preço e quantidade de produto. Esse é um ponto destacado por Lundvall (1988), para quem a interação usuário-produtor é crucial para obter informações qualitativas pertinentes para as atividades inovativas de ambos os partícipes [learning-by-interacting]. Mesmo após a venda do produto ao usuário, o produtor responsabiliza-se ainda por serviços pós-venda referentes à manutenção e soluções de eventuais problemas por seu uso, ajudando a firma compradora na otimização do emprego do bem adquirido.

Uma característica que o conhecimento vem assumindo é a sua crescente complexidade, com forte base nas ciências. Assim o domínio individual das fontes de conhecimento tem se tornado difícil e custoso. Para compreendê-lo e usá-lo de maneira bem-sucedida é necessária a presença e a colaboração de diferentes instituições.

A proximidade geográfica entre produtores e usuários pode ser um requisito valoroso para o aprendizado por interação, dependendo da atividade tecnológica desenvolvida. Lundvall (1988, p. 355) argumenta que para aquelas tecnologias padronizadas e que mudam lentamente, a maior ou menor distância espacial entre os agentes envolvidos não desempenha um papel significativo, dado que a transmissão de informações tem possibilidade de ocorrer em linguagem padrão e a distâncias afastadas. Já naquelas situações em que há mudanças de paradigmas tecnológicos, maior complexidade no conteúdo de informações, ou aceleração do progresso técnico, a proximidade territorial através de contatos face a face e um ambiente cultural compartido assumem função expressiva no sucesso inovativo.

O espaço geográfico compartilhado por agentes econômicos e institucionais tem sido considerado, assim, um fator relevante para a inovação (Gilly; Torre, 2000). Contudo dentre os distintos tipos de proximidade, a geográfica não é a única a influir na capacidade inovativa de empresas e de outras instituições. Boschma (2005) menciona que não se deve dar ênfase apenas à vizinhança espacial e que existe um conjunto de outras proximidades que influem igualmente no aprendizado interativo e que resulta em inovação, tais como a cognitiva, a organizacional, a social e a institucional. ${ }^{5}$

A simples contiguidade geográfica não produz necessariamente a interação entre agentes se os mesmos, em função de racionalidade limitada, apresentem restrições cognitivas, resultando na inexistência de uma base universal de conhecimento que lhes permitam comunicar, absorver e processar informações. A dispersão dos saberes entre diferentes agentes, dadas as especializações, demanda a atuação conjunta para explorá-los de maneira eficaz. Isso, por sua vez, requer um substrato, uma expertise comum sobre a qual possam 5 A síntese realizada nos parágrafos seguintes dos conceitos de proximidade é, com algumas adições, uma interpretação livre do contido no texto de Boschma (2005). 
ocorrer a interação e o aprendizado. A inexistência desses requisitos leva ao isolamento dos agentes, embora próximos espacialmente entre si.

Outro ponto importante a influir no processo de aprendizado e na inovação é o contexto social em que estão inseridos os agentes econômicos ${ }^{6}$. Essa é uma dimensão - desconsiderada pela teoria neoclássica - que influi no comportamento dos agentes e, então, no desempenho econômico e no bem-estar social. Dada a natureza incerta do processo inovativo, mercados atomizados e com atores atuando com racionalidade otimizadora, como supõe o argumento neoclássico, dificilmente levariam à inovação. Para contornar a natureza incerta da inovação e o comportamento não otimizador é necessário o estabelecimento de instituições formais e informais (Maskell e Malmberg, 1999). Assim, quanto mais enraizada estiver a atuação dos participantes, quanto mais intensos forem os laços sociais decorrentes do contato diário - sejam eles de amizade, parentesco, cultural, e outros - mais facilmente são estabelecidas interações para a troca de conhecimentos e a realização de atividades conjuntas, além de permitir a redução de custos de transação. Nesse ambiente, a confiança construída por essas relações, e mesmo aquela decorrente da repetição das trocas comerciais, cria uma espécie de capital, o social, que facilita a difusão de conhecimentos, particularmente os de natureza tácita.

A natureza social da inovação, como assinalada por Doloreux e Parto (2005), deve-se ao fato de o aprendizado e o conhecimento ocorrerem de forma coletiva, seja internamente na empresa através da interação de seus departamentos funcionais, seja por vínculos com agentes externos à firma, como universidades, fornecedores e outros. Igualmente, a proximidade institucional através do compartilhamento de valores éticos, normas, tradições, costumes e regras comuns, sejam essas regras formais ou informais, também contribuem para fortalecer as ligações estabelecidas entre os atores7. Petruzzelli (2008) reporta-se a outro tipo de proximidade: a tecnológica, que se refere a uma base comum de conhecimento ou de similaridade tecnológica entre os participantes da interação, o que facilita o aprendizado e a inovação.

A questão importante colocada por Maskell e Malmberg (1999) é que a globalização tem reduzido a importância para a competitividade daqueles recursos e competências que podem ser facilmente acessados, ou que apresentem caráter ubíquo. O desempenho nos mercados se apresenta com caráter dinâmico, em que o sucesso competitivo de empresas é deslocado para mudanças rápidas em produtos e processos em relação ao que fazem seus competidores. Criar novos conhecimentos, desenvolver atributos dos bens e serviços que sejam particulares às necessidades dos consumidores passam a assumir uma natureza rotineira na vida das firmas. A criação desses

6 Granovetter ([1985] 2001) é o autor de referência sobre o assunto.

7 As proximidades, em suas diferentes dimensões, têm também o poder de inibir o aprendizado e a inovação, na medida em que os agentes fiquem lock-in às práticas e aos conhecimentos existentes. A busca de fontes externas de conhecimento, que não no local, pode ser necessário para incorporar novos saberes e explorar novas trajetórias tecnológicas (Boschma, 2005; Petruzzeli, 2008). 
novos atributos tem ligação com características físicas, econômicas, sociais e institucionais dos locais onde as empresas se localizam. Isto explica porque essas organizações tendem a estabelecer-se em aglomerações produtivas localizadas, em vez de fixarem-se aleatoriamente no espaço geográfico. As externalidades positivas geradas nesses arranjos atraem firmas vinculadas ao setor produtivo dominante e a ramos auxiliares, como observado por Marshall ([1890] 1982), e permitem spin-offs locais.

A capacidade de geração de conhecimentos é resultado de processo de aprendizagem que ocorre durante a vida da empresa. Ao longo do tempo a firma vai aprendendo, entre outros, com seus erros e acertos, pela experiência, por imitação, por esforço deliberado e pela interação com outros agentes. Sob esse aspecto o processo de aprendizado é path-dependence. As capacitações acumuladas pela empresa ao longo do tempo são a base para novas iniciativas. Essa experiência prévia determina, como assinalado por Cohen e Levinthal (1993) a sua 'capacidade de absorção' de novos conhecimentos, principalmente aqueles que são externos a ela.

Igualmente, a atratividade de certas regiões e o grau de desenvolvimento de sua estrutura produtiva depende de evolução histórica. A formação econômica da região está ligada à disponibilidade de recursos que a natureza oferece, da quantidade e da qualificação de mão de obra, de relações sociais, da cultura, e de infraestrutura econômica e institucional construídas. Essa natureza do desenvolvimento há muito foi incorporada à análise econômica através dos economistas históricos alemães. As capacitações existentes na região são fruto não só de seu esforço próprio, mas também daqueles conhecimentos que provêm de fora. Essa combinação reforça características locais e a permanência de diferenças nos níveis de renda entre regiões deve-se a que alguns conhecimentos são específicos a determinados locais, o que lhes conferem alguma vantagem competitiva (Maskell; Malmberg, 1999; Breschi; Malerba, 2001).

Essas particularidades locais nos remetem ao sistema regional ou local de inovação, categoria analítica com nível de amplitude espacial menos abrangente em relação ao sistema nacional de inovação. $\mathrm{O}$ que o conceito tenta referir é o conjunto de atores públicos e privados que mantêm estreitas relações econômicas, sociais, organizacionais, institucionais e outras, com fortes características associadas ao território onde se encontram e que servem como base para o aprendizado, a geração de conhecimento, a inovação e a construção de vantagem competitiva. Obviamente que o conceito requer a identificação da escala territorial apropriada, ou os limites geográficos, a que se aplica (Doloreux; Parto, 2005). Esse é um ponto em que o debate é inconcluso, pois não há homogeneidade em relação ao tratamento dado à região, sendo considerada com extensão variada, ora abrangendo cidades e regiões metropolitanas, até espaços subnacionais, como os estados em países 
federados, por exemplo. ${ }^{8}$ Persistem ainda dúvidas sobre os limites do sistema, ou seja, o que deve ser incluído ou excluído de modo a identificá-lo claramente como unidade de análise (Edquist, 2005).

A dimensão territorial da inovação passou a ganhar notoriedade entre acadêmicos e policy makers a partir do sucesso de aglomerações empresariais localizadas - os chamados distritos industriais - que se observam desde os anos 1970, particularmente a experiência da chamada 'Terceira Itália'. Essa forma de organização industrial caracteriza-se pela presença de um aglomerado de empresas - de um modo geral de pequeno e médio porte - em torno de um setor de atividade e de seus ramos auxiliares, com as empresas inserindo-se no processo produtivo através de uma miúda divisão de trabalho. Além disso, gozam de auxílio de instituições que prestam serviços reais - laboratórios de testes e ensaios, de certificação, de consultoria técnica e gerencial, entre outros - de centros de capacitação da força de trabalho e da presença de universidades e institutos de pesquisa. Becattini (1999) menciona que nos distritos industriais ocorre o imbricamento entre a comunidade local e o conjunto de empresas, o qual é resultado de um processo histórico espontâneo e particular.

Alguns dos agentes desses aglomerados produtivos ou de regiões, conhecidos como knowledge gatekeepers (Petruzzelli, 2008), desempenham um papel de destaque ou de centralidade. Devido à sua capacidade de absorção e de difusão de conhecimentos heterogêneos, como é o caso de universidades e institutos de pesquisa, são capazes de interagir com empresas pertencentes a uma variada gama de atividades econômicas. Essas instituições fazem parte ainda de uma infraestrutura regional mais ampla como meios de transporte e comunicações, equipamentos urbanos, escolas, e arcabouço institucional (Werker; Athreye, 2004).

Releva chamar a atenção ainda de que as inovações apresentam características dos setores em que se desenvolvem (Malerba, 2006). Assim é possível observar diferentes padrões setoriais de inovação. Isso se deve ao fato de que há distinções entre as bases de conhecimento tanto de tecnologias quanto de processos de aprendizagem entre diferentes setores produtivos (Munoz e Encinar, 2008). Setores dedicados a um mesmo ramo produtivo, mas situados em diferentes locais geográficos podem apresentar distintas performances inovativas e competitivas. Parcela do desempenho competitivo, nesses casos, está associada a particularidades regionais ou geográficas, um tipo de capital intangível que não é facilmente clonável.

8 Nesse sentido, o estado brasileiro do Rio Grade do Sul poderia ser tratado como um sistema regional de inovação. Como apontado por Edquist (2005, p. 200): “The choice of approach may not only be a question of size of the country, but also whether it is federally organized or not". 


\section{Informações metodológicas da pesquisa e características da amostra}

Os dados analisados neste estudo foram retirados de duas fontes: uma secundária e outra primária. Os dados de fonte secundária foram coletados junto ao Censo de 2004 do Diretório dos Grupos de Pesquisa do CNPq, que registra, então, os grupos de pesquisa de universidades e as empresas com as quais declararam manter interação. Para o Brasil, naquele ano, foram identificados 2.151 grupos e 1.688 empresas nessa situação.

Os dados primários foram obtidos a partir da realização de uma pesquisa survey, com as empresas que os grupos de pesquisa informaram estabelecer interação. O instrumento utilizado para a coleta de informações constituiu-se de um questionário estruturado. A pesquisa foi realizada em 2009 e recebeu-se um total de 325 questionários, respondidos para todo o País. Para o estado do Rio Grande do Sul, especificamente, o universo foi de 209 empresas, tendo-se recebido 60 questionários.

É importante destacar que a 'amostra' constituída pelas 60 empresas analisadas pode conter vieses, retirando capacidade analítica em direção a generalizações dos resultados encontrados. A resposta ao questionário da pesquisa deu-se por adesão de empresas. O questionário ficou hospedado no site da UFMG, cujo link foi enviado às empresas para o seu acesso e resposta on line. Não houve nesse âmbito interação direta entre pesquisadores e respondentes, de modo que não se soube se ocorreram dúvidas por parte desses últimos em relação às questões formuladas. Contudo, não se pode menosprezar o fato de que alguns itens investigados alcançaram alto índice de respostas semelhantes entre os respondentes, o que fortalece a percepção sobre a importância da proximidade geográfica entre empresas e universidades para a geração e a difusão de inovações.

As informações analisadas neste artigo são, portanto, os da pesquisa survey com as empresas sediadas no Rio Grande do Sul. Para essa análise, em que se busca avaliar o papel da proximidade geográfica na interação entre empresas e universidades, foram utilizadas apenas aquelas perguntas do questionário que estão mais diretamente relacionadas com o tema aqui discutido. Preliminarmente serão fornecidas a seguir algumas características das 60 empresas estudadas e que podem ter influência nas relações estabelecidas ${ }^{9}$.

9 O tratamento dos dados foi feito com apoio do software SPSS e as características da proximidade geográfica foram analisadas com o auxílio do software UCINET. 
A maioria das empresas - um total de 52 de uma amostra de 60 - pertence ao setor industrial, sendo que 27 delas estão envolvidas com as atividades da metalmecânica. Das outras oito, uma é do setor agrícola e sete são do setor de serviços. Quanto ao tamanho das empresas, ${ }^{10} 6$ delas são microempresas, 16 são pequenas, 20 médias e 18 grandes.

As empresas da amostra são predominantemente de propriedade privada, particularmente nacional, pois 51 delas (representando 85,0\% do total) encontravam-se nessa situação. Já as eminentemente estrangeiras são duas e outras duas com essa mesma origem dividem com empresas domésticas o capital aplicado. Há uma empresa pública e uma combinação de quatro outras também públicas em parceria com o capital nacional.

Em relação à localização das empresas da amostra no Estado observa-se que a maior parte (33 empresas) tem suas plantas na Região Metropolitana de Porto Alegre ${ }^{11}$, que inclui cidades do Vale do Sinos, sendo que 13 delas estão localizadas em Porto Alegre e 20 situadas em outros 11 municípios que compõem essa Região. Outra concentração geográfica encontra-se na Serra Gaúcha, com um total de 14 empresas, sendo 11 em Caxias do Sul, uma em Farroupilha, uma em Ipê e uma em Flores da Cunha. As demais 13 empresas estão distribuídas por outras localidades do Estado, com 7 na região Norte, 3 na região Central, 2 na região Sul e 1 na região Nordeste.

\section{Características da proximidade geográfica e da interação entre universidades e institutos de pesquisa com empresas no Rio Grande no Sul}

Das 60 empresas da amostra que informaram manter interação com universidades/institutos de pesquisa, 50 afirmaram ser 'moderadamente importante' ou 'muito importante' a contribuição dessas instituições para as suas atividades de pesquisa nos últimos dez anos. Essas 50 empresas possuíam ao todo um total de 92 interações, ${ }^{12}$ sendo que $67,4 \%$ delas são estabelecidas com instituições no Rio Grande do Sul e 32,6\% com instituições de fora do Estado. A Figura 1 fornece a distribuição desse total de interações alocadas por intervalo de distância geográfica ${ }^{13}$.

\footnotetext{
10 Conforme a seguinte classificação, de acordo com critério do SEBRAE para empresas industriais: microempresa de 1 a 19 empregados; pequeno porte de 20 a 99 empregados; médio porte de 100 a 499 empregados; e grande porte de 500 empregados em diante. E para empresas de comércio e serviços, também segundo o SEBRAE: microempresa até 9 empregados; pequeno porte de 10 a 49 empregados; médio porte de 50 a 99 empregados e grande porte mais de 100 empregados. Disponível em http://www.sebrae.com.br. Acesso em 16.02.2011.

11 De acordo com informações do Atlas Socioeconômico do Rio Grande do Sul (http://www.seplag.rs.gov. br/atlas/atlas.asp?menu=255. Acesso em janeiro de 2011), a Região Metropolitana de Porto Alegre - RMPA, no nordeste do Estado, foi criada por lei em 1973. É composta por 32 municípios que concentram $37 \%$ da população do Rio Grande do Sul.

12 Considera-se neste cálculo as interações estabelecidas com diferentes universidades/institutos de pesquisa, bem como aquelas com diferentes departamentos mesmo que de uma mesma universidade.

13 As informações das distâncias geográficas foram retiradas das respostas à questão 14 do questionário,
} 
FIGURA 1. NÚMERO DE INTERAÇÕES DE EMPRESAS DO RIO GRANDE DO SUL COM UNIVERSIDADES/INSTITUTOS DE PESQUISA, CLASSIFICADOS POR FAIXA DE DISTÂNCIA GEOGRÁFICA EM KILÔMETROS - 2009

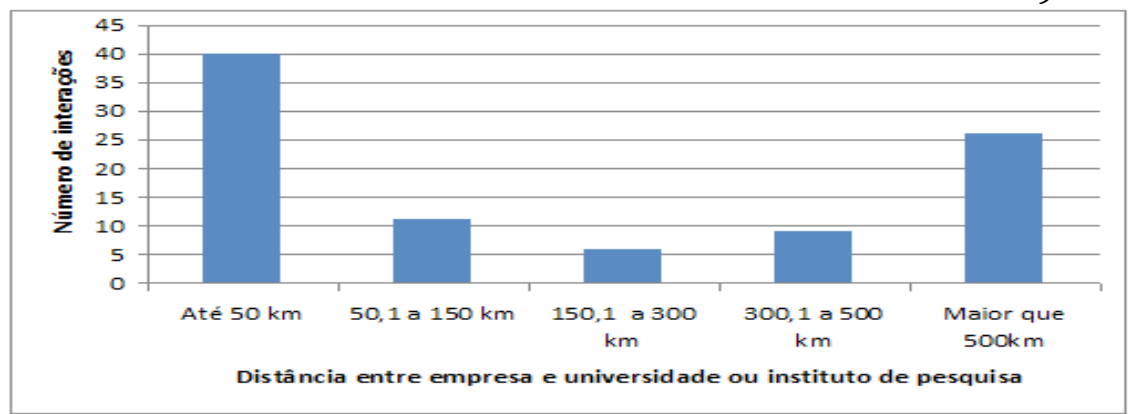

FONTE: BR Survey, 2009.

Do total de 92 interações entre empresas localizadas no estado do Rio Grande do Sul com universidades sediadas no Estado e fora dele, constata-se que $43,5 \%$ ocorriam em menos de $50 \mathrm{~km}$ de distância e mais da metade $(55,5 \%)$ era realizada em um raio de até $150 \mathrm{~km}$. Podem-se considerar essas distâncias como sendo relativamente pequenas ao compararmos com a dimensão geográfica do Estado cuja extensão, segundo informações do Instituto Brasileiro de Geografia e Estatística (IBGE) ${ }^{14}$, é de $268,8 \mathrm{mil} \mathrm{km}^{2}$ : somente o trecho entre Porto Alegre e Caxias do Sul, que representa importante rota da região nordeste do Estado, possui $125 \mathrm{~km}$ de extensão; já para o município de Uruguaiana, essa distância é de $649 \mathrm{~km}$ da capital do Estado.

Verificando no cadastro dos dados da pesquisa a distribuição individualizada dos valores absolutos das distâncias das interações constata-se que os valores da média e do desvio-padrão das distâncias apresentam uma elevada amplitude de dispersão dos dados. A primeira assume o valor de $515,14 \mathrm{~km}$, enquanto o desvio padrão é de $819,10 \mathrm{~km}$, explicado pelos extremos das distâncias de interação, com a mínima de 0,5 km e a máxima de $4.100 \mathrm{~km}$.

O predomínio de interações em até $150 \mathrm{~km}$ distantes dessas instituições entre si pode fundamentar a interpretação de que a proximidade geográfica é um fator facilitador das interações estabelecidas no Rio Grande do Sul. Note-se, ainda, que a maior parte das 50 empresas mantinha vínculos apenas com instituições localizadas no Estado, enquanto somente cinco

\footnotetext{
a qual se apresentava com a seguinte formulação: “Ao longo dos últimos dez anos, qual a importância da contribuição das Universidades ou Institutos de Pesquisa, por área de conhecimento, para as atividades de pesquisa da sua empresa? Indique a Universidade e/ou Instituto de Pesquisa nas áreas que você marcou moderadamente importante (3) ou muito importante (4)". Portanto, as observações aqui descritas e analisadas se referem às interações existentes e consideradas 'moderadamente importantes' e 'muito importantes' pelas empresas. O total de empresas que responderam com quais universidades possuem interação foi de 50 das 60 empresas da amostra. O valor das distâncias em km foi obtido a partir da identificação da distância entre a localização da universidade (campus central) ou do departamento da universidade (quando havia a informação) e a sede da empresa. As informações foram obtidas com auxílio do programa Google Maps.

14 Disponível em http://www.ibge.gov/br/estadosat/perfil.php?sigla=rs. Acesso em 10/02/2011.
} 
delas relacionavam-se somente com instituições situadas em outros estados da Federação, conforme mostra a Tabela 1. Contudo, o fato de empresas se relacionarem com outras instituições que estão sediadas fora do Estado denota que existem outras proximidades, que não a geográfica, que também são importantes para as atividades inovativas das empresas. ${ }^{15}$

TABELA 1. LOCALIZAÇÃO DAS UNIVERSIDADES E INSTITUTOS DE PESQUISA COM AS QUAIS AS EMPRESAS DO RIO GRANDE DO SUL ESTABELECEM INTERAÇÃO - 2009

\begin{tabular}{l|c|c}
\hline \multirow{2}{*}{ Interações } & \multicolumn{2}{c}{ Número de Empresas } \\
\cline { 2 - 3 } & Frequência & \% \\
\hline Somente com instituições do RS & 29 & 58,0 \\
Com instituições do RS e de fora do Estado & 16 & 32,0 \\
Somente com instituições de fora do RS & 5 & 10,0 \\
Total & 50 & 100,0 \\
\hline
\end{tabular}

FONTE: BR Survey, 2009.

A Figura $2^{16}$ a seguir identifica as universidades e outras instituições de pesquisa, bem como as empresas com as quais mantêm interação. As empresas são diferenciadas por números e estão localizadas nas extremidades superiores e inferiores da Figura. As universidades e institutos de pesquisa são distinguidos pelas suas siglas [ver Apêndice] e encontram-se nas extremidades laterais, sendo que à esquerda estão as instituições localizadas no Rio Grande do Sul e à direita as instituições de fora do Estado. Ao todo são 42 instituições, sendo 17 localizadas no Rio Grande do Sul e 25 fora do Estado. ${ }^{17}$ Contudo, embora apresente menor número absoluto de instituições gaúchas nessas interações com as empresas, a intensidade mais acentuada ocorre com elas, pois há maior concentração de linhas que unem os nódulos no lado esquerdo da figura do que no seu lado direito. Os nódulos estão organizados por intensidade de interações: os quadrados azuis representam empresas e universidades/institutos de pesquisa que possuem um número de interações superior aos nódulos em vermelho. Visualmente percebe-se que os agentes que possuem mais interações são em menor número, quando comparados com aquelas empresas e instituições em que as interações embora menos frequentes são em maior número.

\footnotetext{
15 Contudo os dados dos questionários não permitem avançar na discussão sobre o papel de outros tipos de proximidade.

16 Para elaborar as figuras 2,3 e 4 que representam as redes de interações foram incluídas as informações referentes às interações estabelecidas pelas empresas que não responderam à questão 14 do questionário e que constam na base de dados do diretório de grupos de pesquisa do CNPq de 2004. Ao todo são mais 10 empresas nesta situação.

17 Não foram consideradas nesta soma as instituições classificadas como "outra ou estrangeira”, pois se referem àquelas que não foram identificadas no questionário e que poderiam estar localizadas ou não no Rio Grande do Sul.
} 
FIGURA 2. REDE DE INTERAÇÕES ESTABELECIDAS PELAS EMPRESAS COM UNIVERSIDADES E INSTITUIÇÕES DE PESQUISA DO RIO GRANDE DO SUL E DE FORA DO ESTADO - 2009

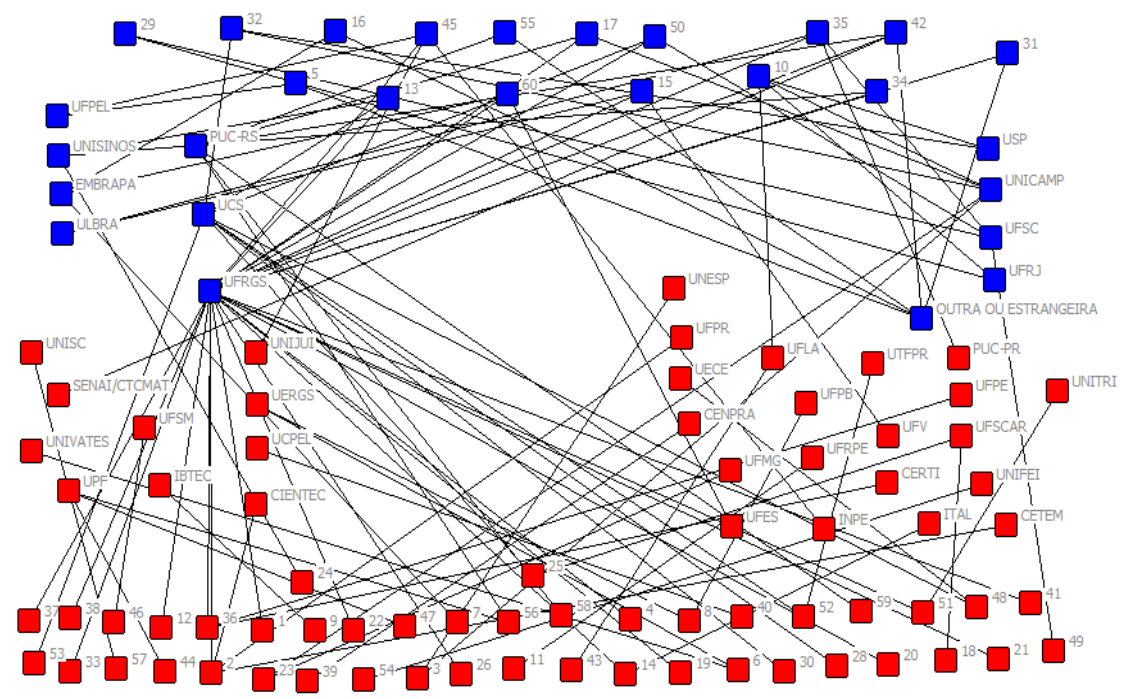

FONTE: BR Survey, 2009.

As universidades gaúchas que se destacam pelo número de interações são a Universidade Federal do Rio Grande do Sul (UFRGS), a Universidade de Caxias do Sul (UCS), e a Pontifícia Universidade Católica (PUC-RS). A UFRGS, principal instituição de ensino e pesquisa do Estado, pode ser vista como desempenhando o papel de knowledge gatekeeper, pois é capaz de interagir com uma diversidade de empresas de diferentes setores produtivos.

As instituições, arroladas como institutos de pesquisa, localizadas no Estado que foram citadas pelas empresas são: a Empresa Brasileira de Pesquisa Agropecuária (Embrapa), o Serviço Nacional de Aprendizagem Industrial (Senai), a Fundação de Ciência e Tecnologia do Rio Grande do Sul (CIENTEC) e o Instituto Brasileiro de Tecnologia do Couro, Calçado e Artefatos (IBTeC).

Em relação às instituições que não estavam localizadas no Rio Grande do Sul merecem destaque duas universidades, pelo número de interações que mantinham com empresas do Estado: a Universidade Estadual de Campinas (Unicamp), em São Paulo, com 10 interações, e a Universidade Federal de Santa Catarina (UFSC), no Estado catarinense, com oito interações. 
Nas Figuras 3 e 4 a seguir as empresas foram organizadas em dois grupos: respectivamente, aquelas que interagiam somente com instituições no Rio Grande do $\mathrm{Sul}^{18}$ e aquelas que mantinham relações com universidades/ institutos de pesquisa pertencentes a outras unidades da Federação.

\section{FIGURA 3. REDES DE INTERAÇÕES ENTRE EMPRESAS COM UNIVERSIDADES E INSTITUTOS DE PESQUISA SOMENTE NO RIO GRANDE DO SUL - 2009}

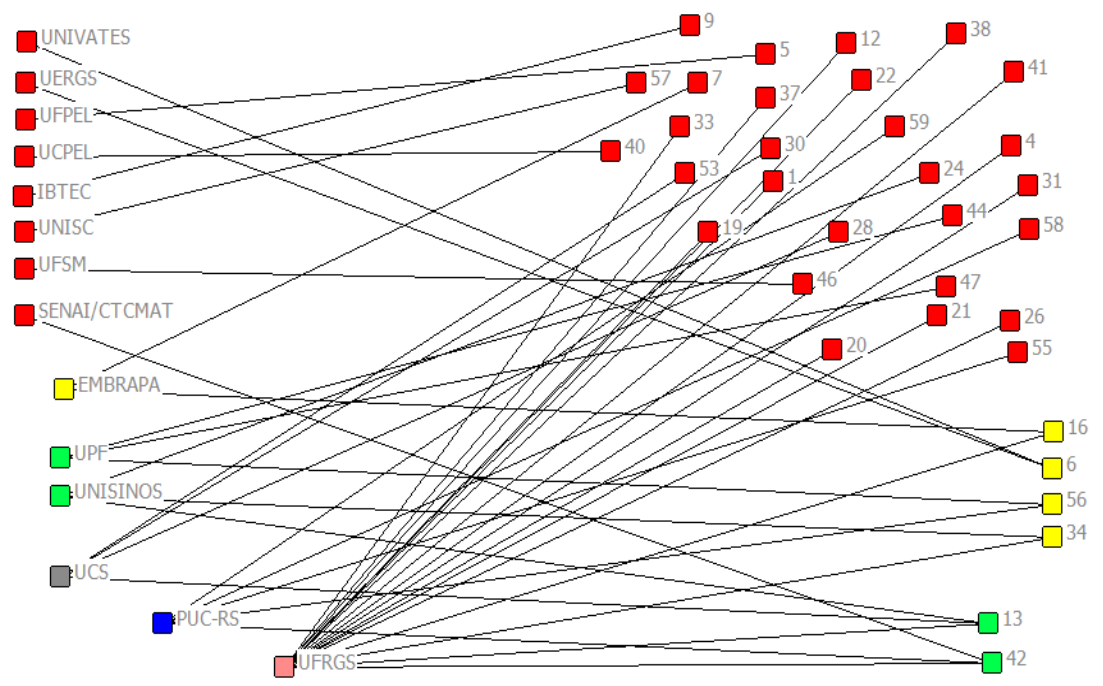

FONTE: BR Survey, 2009.

Os nódulos à direita na figura, identificados por números, representam as empresas e os nódulos à esquerda são as universidades e os institutos de pesquisa do Estado, identificados pelas suas siglas. As cores dos nódulos representam o número de interações existentes: os nódulos vermelhos possuem somente uma interação, os nódulos amarelos possuem duas interações, os verdes três interações, o nódulo cinza quatro interações, o azul cinco interações e o rosa mais de cinco interações. Verifica-se que grande parte das empresas interage apenas com uma universidade ou instituto de pesquisa. Nota-se que a UFRGS e a PUC-RS concentram as interações com as empresas relativamente às demais instituições do Estado, que embora sendo essas últimas em maior número têm menor frequência de relacionamentos. 
FIGURA 4. REDES DE INTERAÇÕES ESTABELECIDAS PELAS EMPRESAS QUE SE RELACIONAM EXCLUSIVAMENTE COM INSTITUIÇÕES DE FORA DO RIO GRANDE DO SUL OU EM SIMULTÂNEO COM OUTRAS DO ESTADO - 2009

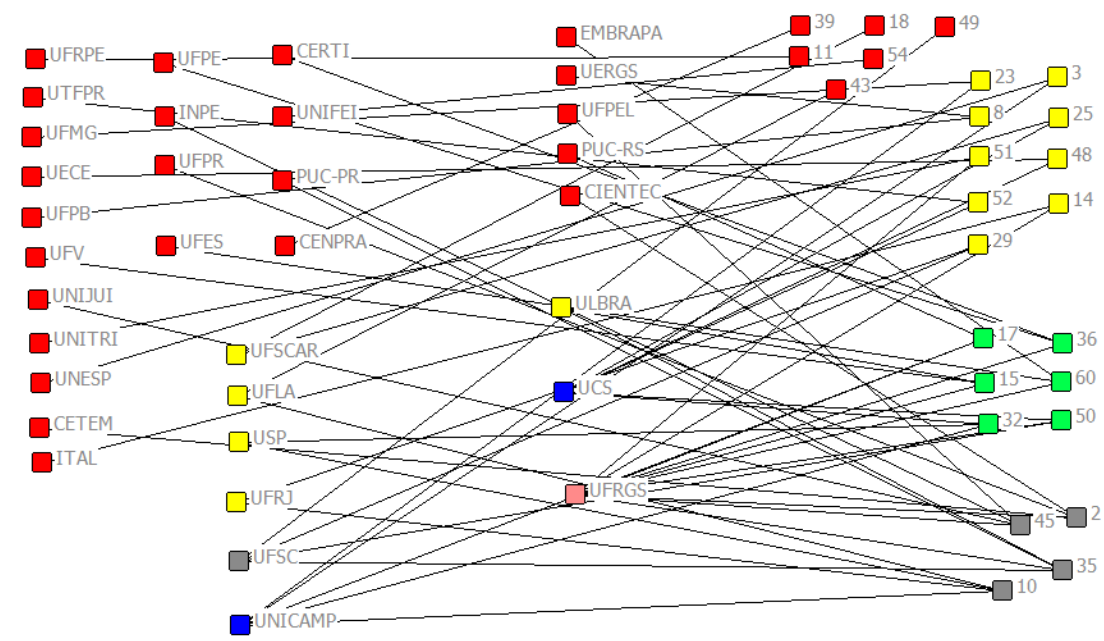

FONTE: BR Survey, 2009.

Na Figura 4 os nódulos estão organizados da seguinte forma: à esquerda estão as universidades e institutos de pesquisa localizados fora do Rio Grande do Sul, no centro as universidades e institutos de pesquisa do Estado e à direita as empresas identificadas por números. As cores dos nódulos representam o número de interações e seguem o mesmo padrão da figura anterior.

Observa-se que há grande diversidade de universidades e instituições de fora do Estado com os quais as empresas interagem. As empresas que estabelecem interações com instituições de fora do Estado, também firmam relações com instituições locais. As universidades e os institutos de pesquisa, localizados além das fronteiras do Estado, com os quais as empresas possuem mais interações, encontram-se principalmente na região Sudeste do país, na UNICAMP, na Universidade Federal do Rio de Janeiro (UFRJ), na Universidade de São Paulo (USP) e na Universidade Federal de São Carlos (UFSCAr).

O tempo de interação das empresas com universidades/institutos de pesquisa é um fator importante a influir nas suas atividades inovativas. O maior tempo de duração dos relacionamentos que as empresas dedicam com as instituições de ensino e pesquisa provavelmente seja resultado de experiências bem-sucedidas em suas necessidades no desenvolvimento de 
produtos, de processos, na solução de problemas do dia a dia, entre outros. Ligações de longo prazo geram confiança, além de proximidades cognitivas, institucionais, e outras, que facilitam a troca de conhecimentos e a sua absorção pelas empresas. Das 50 empresas do Estado que responderam a essa questão, 68,0\% informaram manter interação há mais de cinco anos, sendo que $21,7 \%$ delas interagem há mais de dez anos. Quando se discrimina as empresas pelos setores de atividade, aquelas pertencentes ao setor metalmecânico estão acima da média do Estado. Das 21 empresas desse setor, entre as 27 que responderam o quesito, 80,9\% declararam manter ligação há mais de cinco anos. Esse desempenho deve-se a que o setor metalmecânico tem forte ligação com a área de conhecimento das Engenharias, que como mencionado em outro trabalho (Costa; Ruffoni; Puffal, 2011) é aquela, dentre outras áreas de conhecimento, que figurava no Censo do CNPq de 2004 com maior número de grupos de pesquisa e de empresas com interação no estado do Rio Grande do Sul.

As empresas do Rio Grande do Sul e as do seu setor metalmecânico, em sua maioria, relacionam-se apenas com uma universidade/instituto de pesquisa. No Estado, 38 empresas em 54 responderam que mantêm apenas uma relação, enquanto na metalmecânica esse número era de 13, ou 59,1\% das 22 respondentes, segundo a Tabela 2.

TABELA 2. INTERAÇÕES POR EMPRESA COM UNIVERSIDADES/ INSTITUTOS DE PESQUISA NO RIO GRANDE DO SUL - 2009

\begin{tabular}{|c|c|c|c|c|c|c|c|c|}
\hline \multirow{2}{*}{$\begin{array}{l}\text { Número de } \\
\text { Interações }\end{array}$} & \multicolumn{4}{|c|}{ Rio Grande do Sul } & \multicolumn{4}{|c|}{ Metal-Mecânico } \\
\hline & Freq. & $\%$ & $\begin{array}{c}\% \\
\text { respondentes }\end{array}$ & $\begin{array}{c}\% \\
\text { acum. }\end{array}$ & Freq. & $\%$ & \begin{tabular}{|c|}
$\%$ \\
respondentes
\end{tabular} & $\begin{array}{c}\% \\
\text { acum. }\end{array}$ \\
\hline 1 & 38 & 63,3 & 70,4 & 70,4 & 13 & 48,1 & 59,1 & 59,1 \\
\hline 2 & 7 & 11,7 & 13,0 & 83,4 & 4 & 14,8 & 18,2 & 77,3 \\
\hline 3 & 4 & 6,7 & 7,4 & 90,8 & 3 & 11,1 & 13,6 & 90,9 \\
\hline 4 & 2 & 3,3 & 3,7 & 94,5 & o & 0,0 & 0,0 & 90,9 \\
\hline 5 & 2 & 3,3 & 3,7 & 98,2 & 2 & 7,4 & 9,1 & 100 \\
\hline 8 & 1 & 1,7 & 1,8 & 100,0 & o & 0,0 & 0,0 & \\
\hline Subtotal & 54 & 90 & 100 & & 22 & 81,5 & 100 & \\
\hline $\begin{array}{l}\text { Não } \\
\text { informou }\end{array}$ & 6 & 10,0 & & & 5 & 18,5 & & \\
\hline Total & 60 & 100 & & & 27 & 100 & & \\
\hline
\end{tabular}

FONTE: Diretório dos Grupos de Pesquisa do CNP. Censo de 2004. Elaboração própria. 
As empresas do setor metalmecânico gaúcho interagem relativamente mais com universidades do que as suas congêneres gaúchas que também mantêm relação com aquelas instituições. Enquanto no Rio Grande do Sul apenas 29,6\% das empresas apresentavam mais de uma interação com universidades, no setor metalmecânico esse percentual alcançava 40,9\%. Essa maior intensidade de interação, como já mencionado, pode ter como um de seus determinantes o fato de as empresas envolvidas em atividades do setor metalmecânico terem sua base tecnológica vinculada à área das Engenharias que, junto com as áreas das Ciências Agrárias e das Ciências Exatas e da Terra, são aquelas de maiores graus relativos de interação entre grupos de pesquisa e empresas, entre todas as áreas do conhecimento (Costa; Ruffoni; Puffal, 2011). Essa base comum permite uma maior proximidade tecnológica e cognitiva, pois muitos egressos dos cursos de engenharias têm nas empresas metalúrgicas, mecânicas e outras, as suas futuras ocupações profissionais. Por sua vez, quando no exercício profissional esses egressos, ao se defrontarem com problemas tecnológicos em seu dia a dia recorrem aos laboratórios das universidades e ao conhecimento de ex-professores e de pesquisadores para auxiliá-los na solução. A facilidade de contato face a face pela proximidade geográfica facilita essa interação.

As empresas do Rio Grande do Sul, em número de 16 em 50 respondentes, mantêm vínculos com universidades/institutos de pesquisa localizados no Estado e simultaneamente com instituições que estão fora dessas fronteiras, sendo que outras cinco mantêm exclusivamente relações em outras unidades da Federação. Dessas 21 empresas, nove delas pertenciam ao setor metalmecânico. A Tabela 3 fornece informações sobre esses vínculos, classificando as empresas de acordo com os seguintes atributos: intensidade tecnológica; o setor a que pertence de acordo com a Classificação Nacional de Atividade Econômica - CNAE; a cidade onde está localizada; e o número de interações apresentadas. 
TABELA 3. EMPRESAS QUE MANTÊM INTERAÇÕES COM UNIVERSIDADES/INSTITUTOS DE PESQUISA LOCALIZADOS NO RS E FORA DO ESTADO DO RIO GRANDE DO SUL

\begin{tabular}{|c|c|c|c|c|c|}
\hline & \multirow{2}{*}{$\begin{array}{l}\text { Setor da Empresa } \\
\text { [CNAE 2.0] }\end{array}$} & \multirow{2}{*}{$\begin{array}{c}\text { Intensidade } \\
\text { Tecnológica da } \\
\text { Indústria* }\end{array}$} & \multirow{2}{*}{$\begin{array}{l}\text { Cidade-sede } \\
\text { da empresa }\end{array}$} & \multicolumn{2}{|c|}{$\begin{array}{l}\text { Número de } \\
\text { interações }\end{array}$} \\
\hline & & & & No RS & $\begin{array}{l}\text { Fora } \\
\text { do RS }\end{array}$ \\
\hline \multirow{9}{*}{ 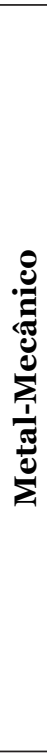 } & $\begin{array}{l}\text { 26.2 Fabricação de equip. de } \\
\text { informática e periféricos }\end{array}$ & Alta tecnologia & Porto Alegre & 2 & a \\
\hline & $\begin{array}{l}\text { 29.4 Fabricação de peças e } \\
\text { acessórios para veículos } \\
\text { automotores }\end{array}$ & $\begin{array}{l}\text { Média-alta } \\
\text { tecnologia }\end{array}$ & Porto Alegre & o & 1 \\
\hline & $\begin{array}{l}29.3 \text { Fabricação de cabines, } \\
\text { corrocerias e reboques para } \\
\text { veículos }\end{array}$ & $\begin{array}{l}\text { Média-alta } \\
\text { tecnologia }\end{array}$ & Caxias do Sul & 1 & 1 \\
\hline & $\begin{array}{l}\text { 29.4 Fabricação de peças e } \\
\text { acessórios para veículos } \\
\text { automotores }\end{array}$ & $\begin{array}{l}\text { Média-alta } \\
\text { tecnologia }\end{array}$ & Caxias do Sul & 2 & 1 \\
\hline & $\begin{array}{l}29.4 \text { Fabricação de peças e } \\
\text { acessórios para veículos auto- } \\
\text { motores }\end{array}$ & $\begin{array}{l}\text { Média-alta } \\
\text { tecnologia }\end{array}$ & Caxias do Sul & 1 & 1 \\
\hline & $\begin{array}{l}\text { 28.3 Fabricação de tratores e } \\
\text { de máq. e equip. para agric. e } \\
\text { pecuária }\end{array}$ & $\begin{array}{l}\text { Média-alta } \\
\text { tecnologia }\end{array}$ & Panambi & 3 & 1 \\
\hline & $\begin{array}{l}\text { 29.4 Fabricação de peças e } \\
\text { acessórios para veículos } \\
\text { automotores }\end{array}$ & $\begin{array}{l}\text { Média-alta } \\
\text { tecnologia }\end{array}$ & Caxias do Sul & 1 & 1 \\
\hline & 24.5 Fundição & $\begin{array}{l}\text { Média-baixa } \\
\text { tecnologia }\end{array}$ & Caxias do Sul & o & 2 \\
\hline & $\begin{array}{l}\text { 25.9 Fabricação de produtos } \\
\text { de metal }\end{array}$ & $\begin{array}{l}\text { Média-baixa } \\
\text { tecnologia }\end{array}$ & Caxias do Sul & 1 & 2 \\
\hline \multirow{9}{*}{ 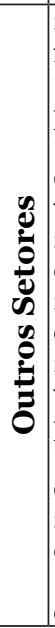 } & $\begin{array}{l}\text { 21.2 Fabricação de produtos } \\
\text { farmacêuticos }\end{array}$ & Alta tecnologia & Porto Alegre & o & 2 \\
\hline & $\begin{array}{l}\text { 15.1 Curtimento e outras } \\
\text { preparações de couro }\end{array}$ & Baixa tecnologia & $\begin{array}{l}\text { Esperança } \\
\text { do Sul }\end{array}$ & 1 & 1 \\
\hline & $\begin{array}{l}\text { 10.3 Fabricação de conservas } \\
\text { de frutas, legumes e outros } \\
\text { vegetais }\end{array}$ & Baixa tecnologia & Farroupilha & o & 2 \\
\hline & $\begin{array}{l}20.3 \text { Fabricação de resinas e } \\
\text { elastômeros }\end{array}$ & $\begin{array}{l}\text { Média-alta } \\
\text { tecnologia }\end{array}$ & Triunfo & 1 & 1 \\
\hline & $\begin{array}{l}\text { 20.1 Fabricação de resinas e } \\
\text { elastômeros }\end{array}$ & $\begin{array}{l}\text { Média-alta } \\
\text { tecnologia }\end{array}$ & Triunfo & 2 & 1 \\
\hline & $\begin{array}{l}20.7 \text { Fabricação de tintas, } \\
\text { vernizes, esmaltes, lacas e } \\
\text { prod. afins }\end{array}$ & $\begin{array}{l}\text { Média-alta } \\
\text { tecnologia }\end{array}$ & $\begin{array}{l}\text { Novo } \\
\text { Hamburgo }\end{array}$ & 2 & 1 \\
\hline & $\begin{array}{l}\text { 19.2 Fabricação de produtos } \\
\text { derivados do petróleo }\end{array}$ & $\begin{array}{l}\text { Média-baixa } \\
\text { tecnologia }\end{array}$ & Canoas & o & 1 \\
\hline & $\begin{array}{l}\text { 22.2 Fabricação de produtos } \\
\text { de borracha }\end{array}$ & $\begin{array}{l}\text { Média-baixa } \\
\text { tecnologia }\end{array}$ & Caxias do Sul & 1 & 1 \\
\hline & $\begin{array}{l}\text { 17.1 Fabricação de celulose e } \\
\text { outras pastas p/ fabr. de papel }\end{array}$ & Baixa tecnologia & Guaíba & o & 4 \\
\hline
\end{tabular}

NOTA: (*) Conforme classificação da OECD (2003). Essa classificação é desenvolvidas para setores industriais. Assim, nesta pesquisa ficaram de fora as empresas pertencentes aos setores 'Extração de carvão mineral', 'Serviços de arquitetura e engenharia' e Geração, transmissão e distribuição de energia elétrica. FONTE: BR Survey, 2009. 
Um ponto a ser destacado nas informações apresentadas nesta Tabela é que essas 21 empresas gaúchas buscam informações e conhecimentos não apenas em instituições de pesquisa no Estado, mas igualmente em universidades/ institutos de pesquisa localizados além de seus limites geográficos. Como a maioria delas mantém igualmente relações com instituições sediadas em solo gaúcho é de se supor que apenas conhecimentos obtidos internamente não atendam às necessidades tecnológicas das empresas. Outro tipo de proximidade, que não a territorial, também é relevante. $O$ fato de a grande parte das empresas estar atuando em atividades classificadas de média-alta tecnologia leva a que se conjecture que alguma expertise ou especialização não está disponível no Estado, ou de que algum tipo de proximidade para obtê-la é mais facilmente desenvolvido com instituições que não estão localizadas em território gaúcho.

Em termos de esforço inovativo, 36 empresas (de 60 respondentes) afirmaram possuir departamento de Pesquisa e Desenvolvimento (P\&D) e 43 (de 52 respondentes) indicaram realizar a prática da inovação de forma regular. As fontes de inovação mais utilizadas pelas empresas para novos projetos e projetos já existentes são principalmente a própria linha de produção e os clientes. Os conhecimentos provenientes das Universidades e dos Institutos, Centros e Laboratórios de Pesquisa também são citados, mas não figuram como os mais utilizados. Entretanto, no que diz respeito à importância das universidades e dos institutos para as atividades de pesquisa das firmas, vale ressaltar, conforme já mencionado, que 50 das 60 empresas afirmaram que as interações com esses atores são 'moderadamente importante' ou 'muito importante'.

Em relação ao papel que a proximidade geográfica, por meio das interações com universidades e institutos de pesquisa, desempenha nas atividades inovativas, constatou-se que grande parte das empresas tem resultados positivos: 24 de 51 responderam que a interação já atingiu os objetivos e 18 afirmaram que o processo ainda está em andamento e os objetivos devem ser atingidos. Somente 6 empresas informaram que a interação não atingiu os objetivos e outras 3 empresas que o processo em andamento não deve apresentar resultados positivos. Outra questão que contribui para a compreensão do desempenho inovativo das empresas refere-se à introdução de produtos e processos no mercado. Para a maioria das empresas as principais inovações são do tipo classificado como aperfeiçoamentos, sejam de produtos ou de processos. Os lançamentos novos, de um ou de outro tipo, para o País ou para o mundo, embora menos frequentes, também são encontrados entre empresas do Rio Grande do Sul. 


\section{Considerações Finais}

O estudo da interação universidade-empresa no Rio Grande do Sul sob a ótica da proximidade geográfica nos permite avançar em algumas reflexões.

As universidades que as empresas gaúchas mais frequentemente se dirigem na busca de informações e conhecimentos para suas atividades inovativas são a UFRGS, junto com outras também de propriedade pública, a Pontifícia Universidade Católica do Rio Grande do Sul (PUC-RS), a Universidade Luterana do Brasil (ULB), a Unisinos, a Universidade de Caixas do Sul (UCS) e outras. Recebe destaque a UFRGS, por ser a universidade com maior número observado de interações com empresas. Pode-se afirmar, inclusive, que algumas dessas universidades, como a UFRGS, desempenham o papel de gatekeeper, pelos variados campos do conhecimento em que mantêm interação com empresas.

As 60 empresas pesquisadas em um universo de 209 são predominantemente de capital privado nacional. Suas escalas são de variados tamanhos, indo do micro ao grande, sobressaindo aqueles de médio porte. Essas empresas, em sua maior parte $(78,3 \%)$, estavam concentradas nas regiões da Grande Porto Alegre (que inclui cidades do Vale do Sinos) e Caxias do Sul. A maioria das empresas - um total de 52 entre as 60 - pertence ao setor industrial, sendo que 27 delas estão envolvidas com as atividades da metalmecânica; as outras oito são dos setores de agricultura, de extração e de serviços.

Como há uma dupla concentração de empresas e de grupos de pesquisa em universidades e institutos localizados nessa região mencionada acima, a interação entre esses agentes ocorre em espaços geográficos relativamente curtos. Essas vizinhanças ganham relevo em um Estado cuja distância rodoviária entre algumas cidades ultrapassa $600 \mathrm{~km}$, sendo inclusive superior aos cerca de $450 \mathrm{~km}$ entre as capitais de Porto Alegre e de Florianópolis, no estado de Santa Catarina, por exemplo. A maior concentração (43,5\%) das 92 interações observadas entre empresas e universidades/institutos de pesquisa ocorre a uma distância de até $50 \mathrm{~km}$, percurso que pode ser percorrido em menos de 60 minutos em uma viagem de automóvel desenvolvendo uma velocidade de $60 \mathrm{~km} / \mathrm{h}$. A maioria $(55,5 \%)$ das interações observadas foi estabelecida a uma distância de até $150 \mathrm{~km}$. Esse fato pode explicar a constatação de que o maior número de interações entre empresas e universidades/institutos de pesquisa ocorre no próprio estado do Rio Grande do Sul. Em 50 das 60 empresas da amostra foram observadas 92 interações com universidades/institutos de pesquisa, cuja importância foi considerada moderada ou de um grau de importância mais elevado para os seus esforços inovativos realizados nos últimos dez anos. Cerca de 2/3 dessas interações ocorreram com instituições sediadas no Rio Grande do Sul. 
Contudo um terço das interações foi realizado com uma gama variada de universidades/institutos de pesquisa em outras unidades da Federação que, inclusive, foram em maior número do que as instituições localizadas no Rio Grande do Sul: 25 e 17, respectivamente. O fato de 21 empresas se relacionarem com instituições que estão localizadas fora do Estado denota que existem outras proximidades, que não apenas a geográfica, que também são importantes para as atividades inovativas das empresas. Como a maioria delas mantém igualmente relações com universidades/institutos de pesquisa do Estado pode-se supor que apenas conhecimentos obtidos no interior das fronteiras do Rio Grande do Sul não sejam suficientes para atender as necessidades tecnológicas das empresas. Outras expertises ou especializações não estão disponíveis no Estado ou, ainda, que algum tipo de proximidade para obtê-las seja mais facilmente desenvolvido com instituições que não estão localizadas em território gaúcho.

O tempo de interação das empresas com universidades e institutos de pesquisa pode ser considerado um indicador da importância que essas instituições representam para as práticas de inovação das empresas. Experiências bem-sucedidas de relacionamentos permanecem no tempo, pois atendem de alguma maneira às necessidades decorrentes do esforço inovativo de empresas. De 50 empresas respondentes, 21 delas informaram manter vínculos com universidades/institutos de pesquisa entre cinco e 10 anos, e 13 mantêm conexões há mais de 10 anos. Quanto ao sucesso desses vínculos, 24 empresas entre 51 responderam que a interação já atingiu os objetivos e 18 que o processo, embora em andamento, teriam os objetivos alcançados.

Quando se classificam as 60 empresas por seus ramos de atividade econômica constata-se que $86,7 \%$ eram do setor industrial. Entre as 52, em torno da metade, ou seja, um número de 27 , estava envolvida na fabricação de produtos da metalmecânica, segmentos industriais de expressão na região entre Porto Alegre e Caxias do Sul. O grau de interação dessas empresas com universidades/institutos de pesquisa é maior do que o das demais empresas. Essa característica observada está associada à base tecnológica por elas empregada, proveniente da área das Engenharias que, dentre outras áreas do conhecimento, apresentava os maiores graus relativos de interação entre grupos de pesquisa e empresas no Rio Grande do Sul.

Em síntese, este esforço de pesquisa para entender o papel desempenhado pela proximidade geográfica entre universidade e institutos de pesquisa com empresas no Rio Grande do Sul permite dar conteúdo à hipótese de que, para essas últimas, a proximidade espacial com essas instituições influi positivamente em suas atividades inovativas. Entretanto, a proximidade geográfica não é a única a exercer influência nesse processo, outras proximidades também devem contribuir para que sejam desenvolvidas inovações nas empresas. A averiguação dessa questão mereceria ser objeto de pesquisas futuras. 


\section{Referências}

BECATTINI, G. (1999). "Os distritos industriais na Itália.” In: GALVÃO, A. \& COCCO, G. \&URANI, A. (orgs.). Empresários e empregos nos novos territórios produtivos: o caso da Terceira Itália. Rio de Janeiro: DP\&A, pp. $45-58$.

BOSCHMA, R. A. (2005). "Proximity and Innovation: a critical assessment." Regional Studies, 39(1): 61-74.

BRESCHI, S. \& FRANCO, M. (2001). "The geography of innovation and economic clustering: some introductory notes." Industrial and Corporate Change, 10(4): 817-833.

COHEN, W. M. \& LEVINTHAL, D. A. (1990). „Absorptive Capacity: a new perspective on learning and innovation." Administrative Science Quarterly, 35: 128-152.

COSTA, A. B. (2009). "Instituições e Competitividade no Arranjo Calçadista do Vale dos Sinos.” Análise Econômica, 27(52): 253-283.

COSTA, A. B.\& RUFFONI, J. \& PUFFAL, D. (2011). “Interação UniversidadeEmpresa no Rio Grande do Sul: o caso do programa de pós-graduação em engenharia de minas, metalúrgica e de materiais da Universidade Federal do Rio Grande do Sul.” In: SUZIGAN, W. \& ALBUQUERQUE, E. \& CARIO, S. F. (orgs.). Em busca da inovação: interação universidade-empresa no Brasil. Belo Horizonte: Autêntica Editora, pp. 199-238.

DOLOREUX, D. \& PARTO, S. (2005). "Regional innovation systems: current discourse and unresolved issues". Technology in Society, 27: 133-153.

EDQUIST, C. (2005). "Systems of innovation: perspectives and challenges." In: FAGERBERG, J. \& MOWERY, D. C. \& NELSON, R. R. (eds). The Oxford Handbook of Innovation. New York: Oxford University Press, pp. 181-208.

FREEMAN, C. (1988). “Japan: a new national system of innovation?" In: DOSI \& FREEMAN, C. \& NELSON, R. \& SILVERBERG, G. \& SOETE, L. (eds.).Technical Change and Economic Theory. London/New York: Pinter Publishers, pp. 330-348.

FUNDAÇÃO DE ECONOMIA E ESTATÍSTICA - FEE. URL [On-line]: $<$ http://www.fee.rs.gov.br>. Acesso em 15 de janeiro de 2011. 
GERTLER, M. S. (2003). "Tacit knowledge and the economic geography of context, or the undefinable tacitness of being (there)." Journal of Economic Geography, 3: 75-99.

GILLY, J. P. \& TORRE, A. (2000). "Proximidad y dinámicas territoriales." In: BOSCHERINI, F. \& POMA, L. (comp.). Territorio, conocimiento y competitividad de las empresas: el rol de las instituciones en el espacio global. Buenos Aires/Madrid: Miño y Dávila Editores, pp.259-294.

GRANOVETTER, M. ([1985] 2001). "Economic Action and Social Structure: The Problem of Embeddedness.” In: GRANOVETTER, M. \& SWEDBERG, R. (eds.). The Sociology of Economic Life. Colorado-EUA/Oxford-UK: Westview Press, pp. 51-76.

LUNDVALL, BENGT-AKE (1988). Innovation as an interactive process: from user-producer interaction to the national system of innovation. In: DOSI, G. \& FREEMAN, C. \& NELSON, R. \& SILVERBERG, G. \& SOETE, L. (eds.). Technical Change and Economic Theory. London/New York: Pinter Publishers, pp. 349-369.

MALERBA, F. (2006). "Innovation and the evolution of industries." Journal of Evolutionary Economics, 16: 3-23.

MARSHALL, A. ([1890] 1982). Princípios de Economia. São Paulo: Abril Cultural.

MASKELL, P. \& MALMBERG, A. (1999). Localised learning and industrial competitiveness." Cambridge Journal of Economics, 23: 167-185.

NELSON, R. R. (1988). "Institution supporting technical change in the United States.” In: DOSI, G. \& FREEMAN, C. \& NELSON, R. \& SILVERBERG, G \& SOETE, L. (eds.). Technical Change and Economic Theory. London/ New York: Pinter Publishers, pp. 312-329.

NELSON, R. R. (ed.). (1993). National Innovation Systems: a comparative analysis. New York: Oxford University Press.

NELSON, R. R. \& Winter, S. G. (1982). An Evolutionary Theory of Economic Change. Cambridge-EUA/London-UK: The Belknap Press of Harvard University Press.

ORGANISATION FOR ECONOMIC CO-OPERATION AND DEVELOPMENT. (2003). Science, Technology and Industry Scoreboard. Paris: OECD. 
PETRUZZELLI, A. M. (2008). "Collaborative knowledge relationships: does proximity matters?" The 25th Celebration Conference on Entrepreneurship and Innovation - Organizations, Institutions, System and Regions, Conpenhagen, CBS, Denmark, Hune 17-20.

PIORE, M. J. (2001). "The emergent role of social intermediaries in the new economy." Annals of Public and Cooperative Economics, 72(3): 339350.

ROSENBERG, N. ([1982] 2006). Por dentro da caixa-preta: tecnologia e economia. Campinas, SP: Editora da Unicamp.

WERKER, C. \& ATHREYE, S. (2004). "Marshall's disciples: knowledge and innovation driving regional economic development and growth." Journal of Evolutionary Economics, 14: 505-523. 
Nome das universidades e institutos de pesquisa citados no texto:

CENPRA: Centro de Pesquisa Renato Archer

CERTI: Fundação Centros de Referências em Tecnologias Inovadoras

CETEM: Centro de Tecnologia Mineral

CIENTEC: Fundação Ciência e Tecnologia do Estado do Rio Grande do Sul

CTCMAT/SENAI: Centro de Tecnologia em Materiais

EMBRAPA: Empresa Brasileira de Pesquisa Agropecuária

IBTEC: Instituto Brasileiro de Tecnologia do Couro, Calçados e Artefatos

INPE: Instituto Nacional de Pesquisas Espaciais

ITAL: Instituto de Tecnologia de Alimentos

PUC/PR: Pontifícia Universidade Católica do Paraná

PUC/RS: Pontifícia Universidade Católica do Rio Grande do Sul

UCPEL: Universidade Católica de Pelotas

UCS: Universidade de Caxias do Sul

UECE: Universidade Estadual do Ceará

UERGS: Universidade Estadual do Rio Grande do Sul

UFES: Universidade Federal do Espírito Santo

UFLA: Universidade Federal de Lavras

UFMG: Universidade Federal de Minas Gerais

UFPB: Universidade Federal da Paraíba

UFPE: Universidade Federal de Pernambuco

UFPEL: Universidade Federal de Pelotas

UFPR: Universidade Federal do Paraná

UFRGS: Universidade Federal do Rio Grande do Sul

UFRJ: Universidade Federal do Rio de Janeiro

UFRPE: Universidade Federal Rural de Pernambuco

UFSC: Universidade Federal de Santa Catarina

UFSCAR: Universidade Federal de São Carlos

UFSM: Universidade Federal de Santa Maria

UFV: Universidade Federal de Viçosa

ULBRA: Universidade Luterana do Brasil

UNESP: Universidade Estadual Paulista Júlio de Mesquita Filho

UNICAMP: Universidade Estadual de Campinas

UNIFEI: Universidade Federal de Itajubá

UNIJUI: Universidade Regional do Noroeste do Est. do Rio Grande do Sul

UNISC: Universidade de Santa Cruz do Sul

UNISINOS: Universidade do Vale do Rio dos Sinos

UNITRI: Centro Universitário do Triângulo

UNIVATES: Centro Universitário Univates

UPF: Universidade de Passo Fundo

USP: Universidade de São Paulo

UTFPR: Universidade Tecnológica Federal do Paraná 